\section{Effects of oral self-care on oral, cognitive, and daily performance functions in rural community-dwelling older people with mild cognitive impairment}

Koji Takada ${ }^{1}$ MS, Chiaki Ura ${ }^{2} \mathrm{PhD}$, Noriko Takei ${ }^{1} \mathrm{MD}$, Kaori Takeda ${ }^{1}$, Seiji Morishima $^{1}$ MS, Takanori Ishii ${ }^{1}$ MS, Masao Ishikawa ${ }^{1}$ MD, Yoko Miyakawa ${ }^{1}$ BD, Fumiko Miyamae ${ }^{2}$ MA, Ryutaro Takahashi ${ }^{2}$ MD, PhD

\begin{abstract}
Background. To determine the effect of an oral self-care programme on oral, cognitive, and daily performance functions in rural communitydwelling older people with mild cognitive impairment.
\end{abstract}

Methods. Community-dwelling older people in 10 villages were assigned based on the village they lived and at the request of the local government to either the intervention group or control group. The oral self-care programme was conducted by a dentist and two dental hygienists twice a month for a total of eight sessions. Oral functions included functions of the lips and cheeks, mastication function, swallowing function, oral diadochokinesis, the longest phonation of 'ah', and cleanliness of the mouth. Cognitive function was assessed using Mini Mental State Examination. The effect of the programme on cognitive function was evaluated using the Digit Symbol Substitution Test (DSST), part A of the Trail-Making Test, and Five Cognitive Tests. Daily performance was assessed using the Tokyo Metropolitan Institute of Gerontology Index of Competence (TMIG-IC).

Results. 32 women in the intervention group and 32 women in the control group were analysed. Intervention had a significant effect on swallowing function in terms of Repetitive Saliva Swallowing Test score $(p<0.001)$ and the longest phonation of 'ah' $(p=0.014)$, cognitive function in terms of the processing speed measured by DSST score $(\mathrm{p}=0.004)$, and daily performance in terms of TMIG-IC score $(\mathrm{p}=0.027)$ and the instrumental independence sub-score $(\mathrm{p}=0.015)$. Surprisingly, mastication power improved significantly in the control group $(\mathrm{p}=0.036)$.

Conclusion. The oral self-care programme may be an effective means to delay oral, physical, and cognitive decline in rural, communitydwelling older people with mild cognitive impairment.

Key words: Aged; Cognition; Independent living; Oral hygiene

\section{ORIGINAL ARTICLE}

The Lion Foundation for Dental Health, Tokyo, Japan

2 Tokyo Metropolitan Institute of Gerontology, Tokyo, Japan
Correspondence to: Koji Takada, The Lion Foundation for Dental Health, 3-7, Honjo 1-Chome, Sumida-ku, Tokyo 130-8644, Japan. Email:kjtakako@circus.ocn.ne.jp 


\section{INTRODUCTION}

In rural Japan, population ageing is an urgent issue. Dementia is of particular concern to communities with limited social and economic resources; effective preventive methods are needed. ${ }^{1}$ Oral hygiene is associated with dementia. The risk of the onset of dementia has been reported to be higher in elderly people who have few teeth and do not use dentures appropriately. ${ }^{2}$ There is a stronger tendency toward a reduced volume of grey matter when the number of teeth decreases. ${ }^{3}$ It has been suggested that once-a-week oral hygiene care by dentists/dental hygienists may suppress the decline in cognitive functions of residents in long-term care facilities. ${ }^{4}$ A comprehensive oral function assessment and oral self-care educational programme have been shown to be effective in improving oral function in longterm care facility residents. ${ }^{5,6}$ This study aimed to evaluate the effects of an oral self-care programme on oral, cognitive, and daily performance functions in rural community-dwelling older people with mild cognitive impairment.

\section{SUBJECTS AND METHODS}

This study was approved by the ethics review board of the Tokyo Metropolitan Institute of Gerontology (Approval No. 24, Kenji No. 964). Informed consent was obtained from each participant. The study was carried out on Miyakojima Island, which is situated approximately $300 \mathrm{~km}$ southwest of Okinawa Island, with a population of about 55,000 (as of 2012). Its population is ageing rapidly, and the proportion of those aged $\geq 65$ years exceeds the average of Okinawa Prefecture (23.2\% vs. $17.4 \%)^{7}$

Between September 2012 and November 2013, community-dwelling older people ( $\geq 65$ years of age) in 10 villages who regularly attended group activities for senior citizens were invited to participate. Subjects were assigned based on the village they lived and at the request of the local government to either the intervention group or control group. Randomisation was not used because (1) most subjects were aged over 75 years and required transportation service; (2) subjects in the same village have always received the same services for administrative purposes; and (3) subjects in the same village would be dissatisfied if they were assigned to a different group from their neighbours.
Dental examination was conducted by one dentist, and oral functions were assessed by two dental hygienists using the method developed by Takei et al. ${ }^{5}$ Functions of the lips and cheeks were evaluated by opening and closing of the mouth and the fullness of the cheeks. Mastication function was based on the degree of dryness of the oral cavity and the mastication power. Swallowing function was based on the Repetitive Saliva Swallowing Test (RSST), oral diadochokinesis in terms of the number of times 'pa', 'ta', and ' $k a$ ' could be pronounced in 5 seconds (reference value, $>31$ ), ${ }^{5}$ and the length of pronouncing ' $a h^{\prime}$ in one breath (reference value, $>15$ seconds). ${ }^{5}$ Cleanliness of the mouth was based on the number of times of tooth-cleaning and the turbidity of spat mouthwash liquid measured by a photoelectrometer (reference value, $0.286 \pm 0.190$ ).

The oral self-care programme ${ }^{5,6}$ was personalised based on the results of oral function tests. Participants were educated twice a month for a total of eight sessions by the dentist and dental hygienists. The perioral (lips and cheeks) muscles were strengthened by increased frequency of conversation, playing wind instruments (e.g. harmonica), and/or singing karaoke. Mastication was strengthened by using both sides of the oral cavity, and salivation was stimulated by brushing or directly massaging the salivary glands. Swallowing was improved by headraising exercises and tongue-holding manoeuvres. Oral cleanliness was enhanced by proper tooth and mucosa brushing.

Cognitive function was assessed using the MiniMental State Examination (MMSE). ${ }^{8}$ The effect of the programme on cognitive function was evaluated using the Digit Symbol Substitution Test (DSST) of the Wechsler Adult Intelligence Scale, ${ }^{9}$ part A of the Trail-Making Test, ${ }^{10}$ and Five Cognitive Tests. ${ }^{11,12}$ The DSST assesses visual motor coordination, attention function, and processing speed. The part $\mathrm{A}$ of the Trail-Making Test assesses visual search speed, scanning, processing speed, mental flexibility, and executive functioning. Five Cognitive Tests assess attention, memory, visuospatial ability, language, and reasoning, based on movements of fingers, character position matching, reproduction of clues, clock drawing, remembering of animal names, and resemblance.

Daily performance was evaluated using the 13- 
item Tokyo Metropolitan Institute of Gerontology Index of Competence (TMIG-IC), ${ }^{13,14}$ which assesses the levels of functional competence higher than those required for activities of daily living. There are three subscales: instrumental independence, intellectual activity, and social roles.

A per-protocol analysis was used, and only subjects who fulfilled all the following criteria were included in the analysis: (1) mild cognitive impairment (MMSE score of $\leq 26$ ), ${ }^{8,15}$ which is more likely to be associated with cognitive decline and physical dependence with ageing ${ }^{16}$; (2) participation in at least six of eight sessions, (3) completion of both baseline and 5-month follow-up assessments, and (4) no missing data.

The intervention and control groups were compared at baseline using the t-test and $\chi^{2}$ test. Analysis of covariance was used to determine group differences after controlling for baseline measures. Age and number of years of education were used as covariates. Independent-sample t-tests indicated that subjects who had missing data did not differ from those who did not with regard to age, educational level, or baseline oral, cognitive, and daily performance measures.

\section{RESULTS}

A total of 22 men and 206 women were recruited.
Subjects in 5 villages were assigned to the intervention group $(n=106)$ and subjects in the other 5 villages were assigned to the control group $(n=122)$. The control group would receive the same oral self-care programme after completion of the study. Respectively in the intervention and control groups, 80 and 82 subjects completed the 5 -month follow-up, of whom 48 and 50 were excluded from analysis because they participated in $<6$ sessions, had a MMSE score of $>27$, or had missing data (Figure).

The remaining 32 women in the intervention group and 32 women in the control group were analysed (TABLE $\mathbf{1}$ ). Their mean age was 82.2 years. Their mean MMSE score was 21.3. 79.7\% of them used dentures regularly. Both groups were comparable in terms of age, the number of years of education, MMSE score, the number of teeth, and the rate of denture use.

Intervention had a significant effect on swallowing function in terms of RSST score $(p<0.001)$ and the longest phonation of ' $a h^{\prime}$ $(p=0.014)$, cognitive function in terms of the processingspeed measured by DSST score $(\mathrm{p}=0.004)$, and daily performance in terms of TMIG-IC score $(\mathrm{p}=0.027)$ and the instrumental independence subscore $(\mathrm{p}=0.015)$ [TABLE 2]. Surprisingly, mastication power improved significantly in the control group $(\mathrm{p}=0.036)$.

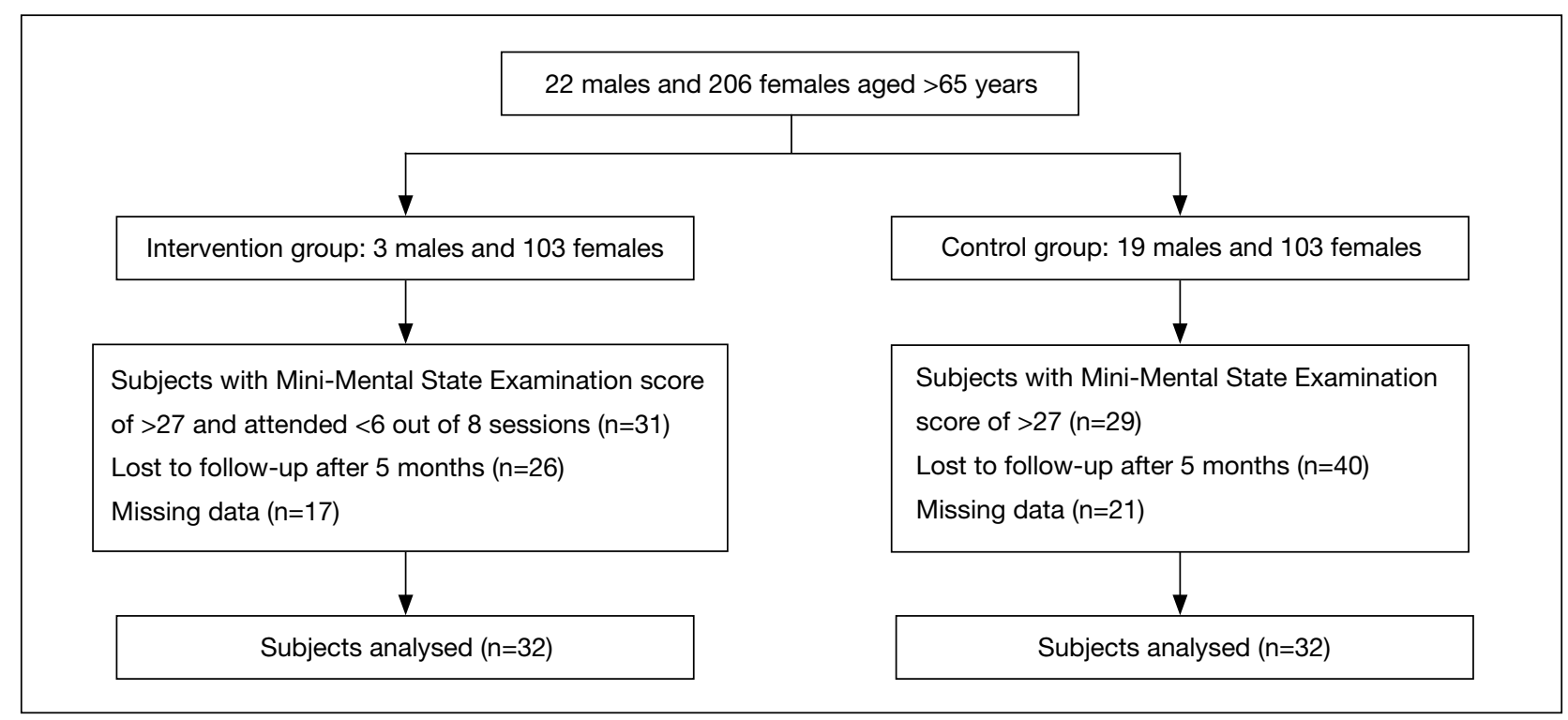

FigurE. Flowchart of subjects 
TABLE 1

Baseline characteristics of participants

\begin{tabular}{lcccc}
\hline Test & Total $(\mathrm{n}=64)$ & Intervention $(\mathrm{n}=32)$ & Control $(\mathrm{n}=32)$ & $\mathrm{p}$ Value \\
\hline Mean \pm SD age (years) & $82.2 \pm 5.0$ & $81.7 \pm 5.2$ & $82.8 \pm 4.8$ & 0.355 \\
Mean \pm SD years of education & $7.1 \pm 2.0$ & $6.9 \pm 2.0$ & $7.3 \pm 2.1$ & 0.541 \\
Mean \pm SD Mini-Mental State Examination score & $21.3 \pm 4.4$ & $21.6 \pm 4.8$ & $21.1 \pm 4.2$ & 0.676 \\
Mean \pm SD No. of remaining teeth & $8.7 \pm 9.8$ & $10.3 \pm 10.0$ & $7.1 \pm 9.0$ & 0.100 \\
No. $(\%)$ of denture wearers & $51(79.7)$ & $26(81.3)$ & $25(78.1)$ & 0.756 \\
\hline
\end{tabular}

TABLE 2

Post hoc analysis of participants

\begin{tabular}{|c|c|c|c|c|c|c|c|c|}
\hline \multirow[t]{2}{*}{ Function } & \multicolumn{2}{|c|}{ Intervention $(\mathrm{n}=32)$} & \multirow[t]{2}{*}{$\mathrm{p}$ Value } & \multicolumn{2}{|c|}{ Control $(n=32)$} & \multirow[t]{2}{*}{$p$ Value } & \multicolumn{2}{|c|}{ Interaction } \\
\hline & Before & After & & Before & After & & $F$ value & $\mathrm{p}$ Value \\
\hline \multicolumn{9}{|l|}{ Oral function } \\
\hline \multicolumn{9}{|l|}{ Lip and buccal area } \\
\hline Opening and closing of oral cavity & $10.0 \pm 0.0$ & $10.0 \pm 0.0$ & - & $10.0 \pm 0.0$ & $10.0 \pm 0.0$ & - & - & - \\
\hline Fullness of the cheeks & $7.8 \pm 3.1$ & $8.8 \pm 2.2$ & 0.099 & $8.3 \pm 3.0$ & $8.8 \pm 2.2$ & 0.500 & 0.490 & 0.487 \\
\hline \multicolumn{9}{|l|}{ Mastication function } \\
\hline Degree of saliva wetness & $2.2 \pm 1.8$ & $2.6 \pm 1.5$ & 0.223 & $2.1 \pm 1.4$ & $2.4 \pm 2.9$ & 0.485 & 0.138 & 0.711 \\
\hline Mastication power & $2.6 \pm 0.7$ & $2.8 \pm 0.9$ & 0.101 & $1.8 \pm 0.7$ & $2.2 \pm 0.8$ & $<0.001$ & 4.618 & 0.036 \\
\hline \multicolumn{9}{|l|}{ Swallowing function } \\
\hline Repetitive Saliva Swallowing Test & $1.5 \pm 1.1$ & $2.8 \pm 0.8$ & $<0.001$ & $1.8 \pm 1.1$ & $1.7 \pm 1.0$ & 0.733 & 25.168 & $<0.001$ \\
\hline Oral diadochokinesis 'ka' (no./5 sec) & $25.5 \pm 3.9$ & $26.9 \pm 2.7$ & 0.018 & $24.4 \pm 4.4$ & $24.7 \pm 3.6$ & 0.879 & 2.580 & 0.114 \\
\hline Longest phonation of 'ah' (sec.) & $13.9 \pm 4.4$ & $16.5 \pm 4.4$ & $<0.001$ & $12.1 \pm 3.7$ & $14.4 \pm 5.0$ & 0.732 & 6.367 & 0.014 \\
\hline \multicolumn{9}{|l|}{ Oral cleanliness } \\
\hline Score in no. of times of tooth-cleaning & $6.4 \pm 2.9$ & $8.3 \pm 2.4$ & $<0.001$ & $5.0 \pm 3.8$ & $6.4 \pm 3.4$ & 0.021 & 0.971 & 0.328 \\
\hline Turbidity of spat mouthwash liquid & $0.386 \pm 0.301$ & $0.277 \pm 0.174$ & 0.009 & $0.479 \pm 0.290$ & $0.387 \pm 0.270$ & 0.023 & 0.062 & 0.805 \\
\hline \multicolumn{9}{|l|}{ Cognitive function } \\
\hline Mini-Mental State Examination & $21.6 \pm 4.8$ & $23.3 \pm 5.4$ & 0.011 & $21.1 \pm 4.2$ & $22.9 \pm 4.6$ & 0.004 & 0.055 & 0.815 \\
\hline Digit Symbol Substitution Test & $22.0 \pm 10.7$ & $24.6 \pm 12.0$ & 0.006 & $20.6 \pm 8.8$ & $19.3 \pm 8.6$ & 0.157 & 9.097 & 0.004 \\
\hline Part A of Trail-Making test & $112.0 \pm 59.2$ & $108.4 \pm 56.0$ & 0.768 & $123.3 \pm 53.2$ & $111.2 \pm 50.6$ & 0.180 & 0.529 & 0.470 \\
\hline \multicolumn{9}{|l|}{ Five Cognitive Tests } \\
\hline Movements of fingers & $12.4 \pm 4.9$ & $14.7 \pm 5.2$ & 0.005 & $11.3 \pm 4.5$ & $13.1 \pm 5.4$ & 0.023 & 0.168 & 0.683 \\
\hline Character position matching & $6.7 \pm 5.2$ & $8.2 \pm 5.6$ & 0.050 & $4.8 \pm 5.3$ & $4.7 \pm 4.7$ & 0.998 & 1.985 & 0.164 \\
\hline Reproduction of clues & $6.0 \pm 4.1$ & $6.9 \pm 4.7$ & 0.009 & $4.0 \pm 2.8$ & $4.9 \pm 3.3$ & 0.006 & 0.010 & 0.921 \\
\hline Clock drawing & $5.1 \pm 2.4$ & $4.8 \pm 2.4$ & 0.388 & $4.8 \pm 2.3$ & $4.7 \pm 2.3$ & 0.741 & 0.143 & 0.707 \\
\hline Remembering of animal name & $8.0 \pm 3.2$ & $8.7 \pm 3.0$ & 0.137 & $6.8 \pm 2.6$ & $7.4 \pm 2.2$ & 0.126 & 0.001 & 0.976 \\
\hline Resemblance & $2.3 \pm 2.2$ & $2.4 \pm 2.7$ & 0.753 & $1.4 \pm 2.2$ & $1.5 \pm 2.3$ & 0.686 & 0.004 & 0.950 \\
\hline \multicolumn{9}{|l|}{ Daily performance } \\
\hline $\begin{array}{l}\text { Tokyo Metropolitan Institute of } \\
\text { Gerontology Index of Competence }\end{array}$ & $10.4 \pm 2.7$ & $10.9 \pm 2.4$ & 0.248 & $10.6 \pm 2.2$ & $9.8 \pm 2.6$ & 0.043 & 5.169 & 0.027 \\
\hline Instrumental independence & $4.0 \pm 1.5$ & $4.2 \pm 1.3$ & 0.581 & $4.1 \pm 1.3$ & $3.4 \pm 1.5$ & 0.004 & 6.337 & 0.015 \\
\hline Intellectual activity & $3.1 \pm 0.9$ & $3.2 \pm 0.9$ & 0.551 & $2.8 \pm 1.2$ & $2.9 \pm 1.1$ & 0.510 & 0.002 & 0.965 \\
\hline Social role & $3.3 \pm 1.1$ & $3.5 \pm 0.8$ & 0.194 & $3.7 \pm 0.5$ & $3.4 \pm 0.8$ & 0.140 & 3.902 & 0.053 \\
\hline
\end{tabular}




\section{DISCUSSION}

In a preliminary study, the oral self-care programme tended to delay cognitive decline in residents in a group home. ${ }^{6}$ In our study, the DSST score at 5 months was significantly higher in the intervention than control group; the oral self-care programme may have had a positive effect on visual motor coordination, attention, and processing speed. The DSST may be important in the understanding of age-related decline of cognitive functions. ${ }^{17}$ In addition, a low DSST score alone or combined with a slow gait has been reported to be associated with mortality and development of disability, independent of other risk factors. ${ }^{18}$ Thus, significant improvement in the DSST score after the oral selfcare programme suggests an association between the delay in cognitive and functional decline and the programme. Furthermore, the significant effect of the programme on swallowing function suggests an association between the programme and improved eating and speaking ability, both of which are crucial in maintaining good health, daily performance, and social contact. Improvement in instrumental independence and daily performance may also delay physical and cognitive decline.

There was an unexpected significant improvement in mastication power in the control group. This may be because the control group had a lower baseline value (1.8 vs. 2.6). The Hawthorne effect (i.e. novelty of being research subjects and the increased attention from that) could also lead to a temporary increase in performance. ${ }^{19}$

Caution should be taken in making generalisation about our findings. The subjects rarely leave the island and have strong ties in the local community. Randomisation was not done. About two thirds of the subjects dropped out or were excluded from analysis. The subjects might not have been familiar with receiving any programme or assessment and might have been confused about the objective of the study, and thus did not attend the follow-up assessment at 5 months. In addition, the time of the study coincided with that for harvesting sugar cane and religious family commemorations and celebrations. These may have interfered with the intervention sessions and/or follow-up assessment. The proportion of male participants was small owing to cultural and practical factors. In Japan, most elderly people registered at day centres are female. Japanese men are less motivated to engage in preventive health care. To reduce the dropout rate, more individual motivation to continue participation until follow-up assessment and careful timing of the study to avoid interference by planting or harvesting are needed.

\section{CONCLUSION}

The oral self-care programme may be an effective means to delay oral, physical, and cognitive decline in rural, community-dwelling older people with mild cognitive impairment.

\section{ACKNOWLEDGEMENTS}

The authors thank Kyoko Tomiyama, Kyoko Nema, Misako Nakasone, and all other staff in the Miyakojima Public Office for facilitating this study. The authors also thank nurses Kazumi Takeshima, Chiyoko Oshiro, Setsuko Taira, Takako Taira, Matsue Kugai, and dental hygienists Saori Maesato and Junko Shimosato for their cooperation. We also express our appreciation to all participants.

This study was supported in part by a Grantin-Aid for Scientific Research (H22-NinchishoIppan-004) from the Ministry of Health, Labor and Welfare of Japan, and in part by a Grant-in-Aid for Scientific Research from the Ministry of Education, Science, Sports, Culture and Technology, Japan (24659940).

\section{REFERENCES}

1. WakutaniY, Kusumi M, Wada K, et al. Longitudinal changes in the prevalence of dementia in a Japanese rural area. Psychogeriatrics 2007;7:150-4.

2. Yamamoto T, Kondo K, Hirai H, Nakade M, Aida J, Hirata Y. Association between self-reported dental health status and onset of dementia: a 4-year prospective cohort study of older Japanese adults from the Aichi Gerontological Evaluation Study (AGES) Project. Psychosom Med 2012;74:241-8.

3. Watanabe $\mathrm{M}$. The relationship between oral health and brain function [in Japanese]. J Jpn Dent Assoc 2009;28:60-3.

4. Kikutani T, Yoneyama T, Nishiwaki K, Tamura F, Yoshida M, Sasaki H. Effect of oral care on cognitive function in patients with dementia. Geriatr Gerontol Int 2010;10:327-8.

5. Takei N, Fujimoto A, Kimoto E, et al. A study of unified system of assessment and control of oral function for the elderly. First report: a comprehensive method of examination and improvement, and result evaluation for the independent elderly [in Japanese]. Jpn J Gerodontol 2009;23:384-96.

6. Ishikawa M, Takei N, Ishii $\mathrm{T}$, Takada $\mathrm{K}$, Hamada $\mathrm{M}$. The effect of an oral function improvement program on the cognitive function 
of the elderly in a group home [in Japanese]. Jpn J Gerodontol 2015;30:37-45.

7. Japan Medical Association. Japan Medical Analysis Platform. Available from: http://jmap.jp/cities/detail/city/47214/. Accessed 16 May 2016.

8. Folstein MF, Folstein SE, McHugh PR. "Mini-mental state". A practical method for grading the cognitive state of patients for the clinician. J Psychiatr Res 1975;12:189-98.

9. Wechsler D. Wechsler Adult Intelligence Scale-Revised. San Antonio: Psychological Corporation; 1981.

10. Lezak MD. Neuropsychological assessment. 3rd ed. New York: Oxford University Press; 1995.

11. Sugiyama M, Ijuin M, Sakuma N, et al. Reliability and validity of the five cognitive test in the context of detecting older people with mild cognitive impairment living in the community [in Japanese]. Jpn J Geriatr Psychiatry 2015;26:183-95.

12. Maki $Y$, Ura C, Yamaguchi T, et al. Effects of intervention using a community-based walking program for prevention of mental decline: a randomized controlled trial. J Am Geriatr Soc 2012;60:505-10.

13. Koyano W, Shibata H, Nakazato K, Haga H, Suyama Y.
Measurement of competence: reliability and validity of the TMIG Index of Competence. Arch Gerontol Geriatr 1991;13:10316.

14. Seino S, Shinkai S, Fujiwara $Y$, et al. Reference values and age and sex differences in physical performance measures for community-dwelling older Japanese: a pooled analysis of six cohort studies. PLoS One 2014;9:e99487.

15. O'Bryant SE, Humphreys JD, Smith GE, et al. Detecting dementia with the mini-mental state examination in highly educated individuals. Arch Neurol 2008;65:963-7.

16. Sun F, Norman IJ, While AE. Physical activity in older people: a systematic review. BMC Public Health 2013;13:449.

17. Salthouse TA. What do adult age differences in the Digit Symbol Substitution Test reflect? J Gerontol 1992;47:121-8.

18. Rosano C, Newman AB, Katz R, Hirsch $\mathrm{CH}$, Kuller LH. Association between lower digit symbol substitution test score and slower gait and greater risk of mortality and of developing incident disability in well-functioning older adults. J Am Geriatr Soc 2008;56:1618-25.

19. Mayo E. The social problems of an industrial civilization. Boston: Division of Research, Harvard Business School; 1945:161-82. 